\title{
USER SELECTION CRITERIA OF AIRSPACE DESIGNS IN FLEXIBLE AIRSPACE MANAGEMENT
}

\author{
Hwasoo E. Lee, Paul U. Lee, San Jose State University, San Jose, CA \\ Jaewoo Jung, Chok Fung Lai, University of California, Santa Cruz, CA
}

\begin{abstract}
Flexible Airspace Management (FAM) concept offers to dynamically modify the center/sector boundaries in such way that the airspace structure is reconfigured to better distribute unbalanced traffic demands across sectors. A set of airspace design algorithms were used in the human-in-the-loop simulation to assess possible benefits of the FAM concept. In the simulation, participants were instructed to pick an algorithm-generated airspace configuration from a set of configuration options that best solved the weather-induced traffic imbalance problems in the test airspace. Participants also rated the acceptability of the airspace designs that were generated by different algorithms. This paper explores ways to objectively quantify airspace characteristics of these algorithm-generated configurations using a set of benefits and airspace quality metrics and to compare them to the participants' acceptability ratings obtained from the simulation. Both benefits and airspace quality metrics were hypothesized to correlate with the participants' ratings. The results showed that participants' selection correlated mainly with the benefits metrics, while airspace quality metrics did not play a big role.
\end{abstract}

\section{Introduction}

The National Airspace System is often challenged by problems arising from imbalance between traffic load demand and air traffic control capacity. Within the framework of the Next Generation Air Transportation System (NextGen), one of the proposed solutions to these problems is the Flexible Airspace Management (FAM) concept (previously known as Dynamic Airspace Configuration) [1]. FAM offers to dynamically modify the center/sector boundaries in such way that the airspace structure is reconfigured to better distribute unbalanced traffic demands across sectors, thereby reducing the need to implement various flow restrictions.

A human-in-the-loop simulation was conducted in 2010 [2,3] in the Airspace Operations Laboratory at NASA Ames Research Center to test the FAM concept, as a follow up to a previous study [4]. It comprised participants choosing airspace configuration from a set of algorithm-generated ones that best solved traffic imbalance problems due to weather deviation. The study was successful in assessing the potential benefits of FAM and its implications to the roles and responsibilities of various players involved in FAM operations, as well as exploring, among other things, the potential role of airspace optimization algorithms in the airspace reconfiguration portion of FAM operations. However, past analyses did not fully explore the relationship between the airspace characteristics and its role in the user selection of the preferred airspace designs. Hence, this paper explores this relationship using a set of metrics used by Jung et al. [5].

This paper is organized as follows. FAM section provides some background information of the study as well as a short description of the algorithms used in the study. The Metrics section describes the metrics used to quantify airspace characteristics. A summary of results are presented in the Result section and the paper ends with the Discussion section.

\section{Flexible Airspace Management}

Although an extensive description of the FAM concept and its human-in-the-loop study conducted in $2010[2,3]$ are beyond the scope of this paper, this section includes a brief explanation of the study to provide the context of the airspace and the operational environment for the study.

\section{Participants and Airspace}

Four participants from the FAA with Traffic Management Coordinator and/or Front Line Manager experience were recruited for the simulation. They were presented with four weather-induced sector load imbalance problems involving four or seven sectors in Kansas City Center (ZKC), depending on the 
scope of the traffic problem. These sectors were grouped into one and two areas of specialization (AOS) compare FAM operations in both a relatively simple (4-sector within one AOS) and a more complex (7-sector within two AOSs) environment. The four test sectors (ZKC sectors 28, 29, 30, and 92) and the seven test sectors (ZKC sectors 3, 28, 29, 30, 47, 92, and 94) were adapted from high altitude sectors in ZKC (Figure 1).

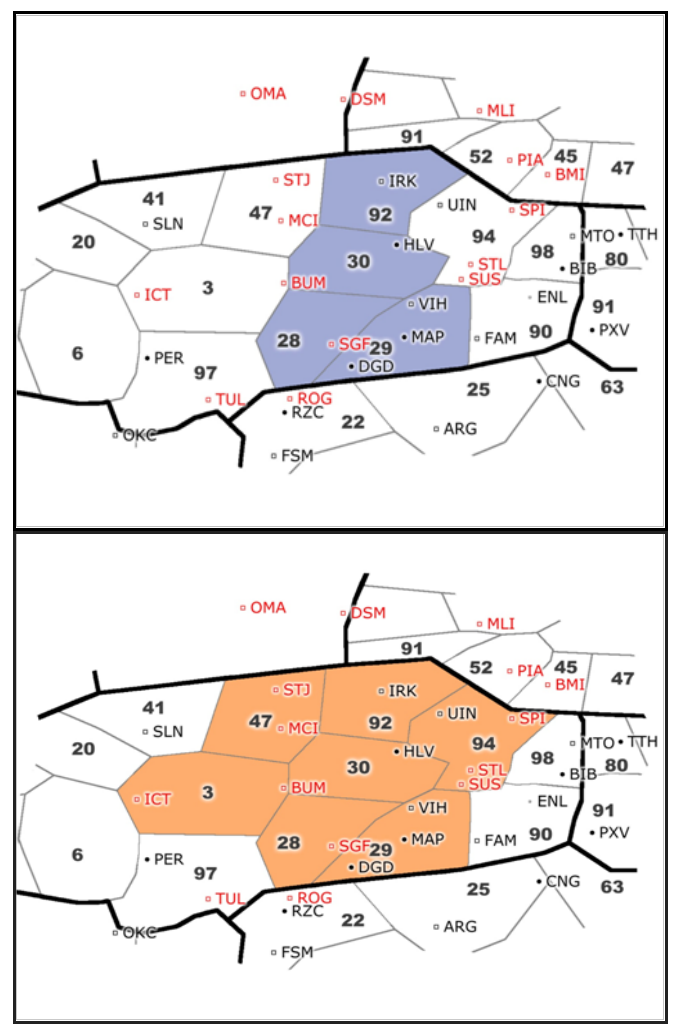

\section{Figure 1: Test Sectors for the 4-Sector (Top) and 7-Sector (Bottom) Traffic Scenarios}

\section{Traffic Scenarios}

Traffic scenarios were developed using the Multi-Aircraft Control System's (MACS) scenario editor function [6]. After determining the set of ZKC sectors that would be used, a base set of traffic was developed for each of the 4- and 7-sector traffic scenarios. The initial effort involved generating traffic loads that ranged between sustained counts of 15 and 22 aircraft in each of the test sectors over time to mimic the nominal operation environment. Then, convective weather cells were developed and then integrated with the traffic scenarios which were then modified by a pair of recently retired controllers in such way that the traffic is rerouted around the weather cells, but kept within the test airspace to avoid reducing the overall traffic demand. As a result, some sectors significantly exceeded the maximum 22-aircraft sector load capacity. Traffic overload usually occurred after 45 minutes into the simulation runs. The resulting traffic data were used to generate airspace configurations according to different algorithmic approaches that tried to minimize the traffic overload in the test sectors while maintaining "good" airspace design characteristics whenever possible. Details of the algorithmic approaches explained in a subsequent section.

\section{Procedure}

Although the experiment consisted of a $3 \times 2$ within-subjects design with two factors, namely the boundary change condition, and the number of sectors involved in the reconfiguration (i.e., four or seven sectors), this paper discusses one of the three boundary change conditions, in which the participants were given a list of pre-defined airspace configuration options generated by the aforementioned algorithms to determine which option was the best candidate for the given traffic scenario. In particular, the participants were given traffic load graphs that showed traffic demand in 15-minute intervals, up to 2.5 hours in future, and were asked to select an airspace design that would best solve the traffic overload problem and at the same time result in airspace configuration change that is workable by the controllers.

The traffic load graph showed impacts of different airspace designs on the number of aircraft in each sector during the traffic scenario. Therefore, the airspace configuration selection process performed by the participants was based on a prediction of the traffic loads within the test airspace. Once the selection was done, they could further modify the selected configuration manually if desired, but this paper's scope is limited only to the selection part-task.

\section{Algorithms}

An important objective of this study was to explore the role and efficacy of airspace optimization algorithms in airspace design. To examine this question, four algorithmic approaches were used to design airspace configuration sets according to their unique 
design criteria. These four approaches are described in the following paragraphs.

\section{Dynamic Airspace Units (DAUs)}

The first approach initially partitions portions of the airspace into what are called Dynamic Airspace Units (DAU) through a series of incremental slices between neighboring sectors. These units are assigned to the appropriate sector(s) based on the most effective distribution of traffic demand within the defined airspace. As the distribution of traffic changes over time, new sets of sectorizations can be generated at defined intervals to reflect the changes and reduce the instances of sectors being over- or under-loaded [7].

\section{CellGeoSect (CGS)}

A second approach combines two separate algorithms to arrive at its design. It first uses Mixed Integer Programming to balance a number of metrics, such as dwell time and aircraft count imbalance between sectors [8]. It then divides the airspace into a network of small hexagonal cells and systematically combines the adjacent cells that share common traffic flows while maintaining the optimization criteria of balancing traffic. Once this approach arrives at an airspace design, the resultant airspace configuration is then fed into a Binary Space Partition algorithm that can incorporate air traffic operational constraints related to sector shapes and critical flow intersection points directly into the model to arrive at an airspace design that meets the operational needs [9].

\section{SectorFlow (SF)}

A third approach creates sector boundaries first by clustering time-sampled aircraft positions together according to defined clustering criteria in order to capture flows through a given airspace. This clustering of positions is further refined through region growing methods that fill the empty regions between clusters by assigning the remaining aircraft positions to the appropriate clusters. Based on the resulting cluster profile, computational geometry techniques are applied to form the initial airspace boundary configuration that most efficiently encloses the aircraft positions in each cluster for a given time period. Once established, the boundary configurations are adjusted to balance Dynamic Density (DD) factors throughout the airspace while minimizing the impact of the configuration on user-preferred flight routings [10]. DD factors refer to a set of metrics that are correlated with traffic complexity and can be more accurate predictors of workload than traditional aircraft count alone [11].

\section{Modified Voronoi (MV)}

A fourth and final approach uses a combination of Voronoi diagrams and genetic algorithms to optimize the airspace design [12,13]. Voronoi diagrams are used to initially partition the airspace into convex-shaped sectors that have an associated set of "generating points." Genetic algorithms are then used to optimally configure those points into an airspace design that minimizes a set of predefined cost metrics (e.g., aircraft count, flight dwell time, number of sector boundary crossings, etc.). Further consideration of the cost metrics is given in the design to avoid positioning boundaries in close proximity to traffic intersection areas. An iterative deepening method was also used for the designs in this study to allow for the vertical partitioning of airspace and the ability to define and maintain the number of sectors required for the final configuration. This deepening method "searches" through a defined depth - the airspace floor in this case - for the solution that best meets the end-state goals. This was a necessary addition that allowed for reconfiguration options in both the lateral and vertical dimensions, as the previous study was only limited to the lateral dimension.

Figure 2 below illustrates a set of airspace designs created by the four algorithmic approaches for one of the 4-sector traffic scenarios.

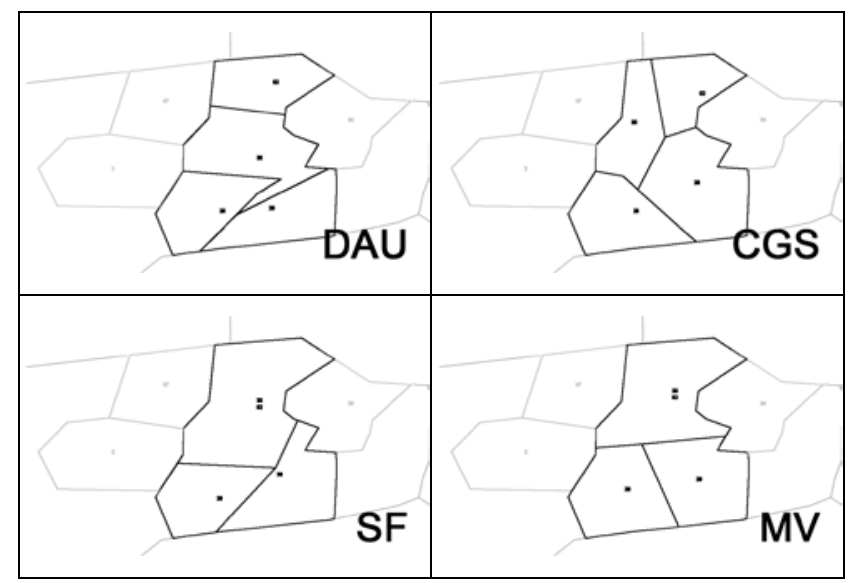

Figure 2: Four Examples of Algorithm-Generated Airspace Designs for a 4-Sector Traffic Scenario (N.B. MV has an airspace configuration that first combined sectors and then split the sectors vertically by altitudes.) 


\section{Metrics}

Of particular interest in the FAM study was to compare participants' ratings on the algorithmgenerated configuration that was best suited for the given traffic scenario, and to find out the airspace characteristics that influenced their selection. For that reason, in this paper, these four algorithms are quantified using a set of metrics used by Jung et al. [5]. The metrics are divided into two main categories: benefits and airspace quality metrics. The benefits metrics included the number of flights that needed to be removed to reduce the peak aircraft count in any sector to 22 aircraft or less, and the percentage of air traffic control capacity that is utilized in each test sector. The airspace quality metrics included the airspace similarity between the original and the final airspace configuration, the number of flights with short dwell time, and the average distance between traffic crossing points and the nearest airspace boundary.

These metrics were computed using Airspace Concept Evaluation System (ACES) [14] and are described in the following subsections.

\section{Benefits Metrics}

\section{Number of Removed Aircraft}

Weather reroutes effectively shift traffic demand in airspace from severe weather regions to the remaining areas. If this shift resulted in a mismatch between demand and capacity of the affected areas, the number of flights would need to be reduced by delaying and/or turning away those that enter these affected areas to reduce the demand, and hence, to rebalance the loads.

Let $F_{i}$ be the number of aircraft removed to balance demand and capacity in airspace configuration $i$. To calculate $F_{i}$, first, the sector with the highest peak aircraft count in the scenario is identified. Then, this sector's peak aircraft count is compared to the default sector capacity of 22 aircraft. If the peak count is larger than the threshold, the flight that dwells the longest in the identified sector is removed from the airspace configuration $i$ to reduce the demand, and $F_{i}$ is increased by one. Thereafter this process repeats with the reduced demand. If the peak count is the same or less then the threshold, calculation of $F_{i}$ is completed. $F_{i}$ is initially set to zero.

\section{Air Traffic Control Resource Utilization}

The ratio of an average sector aircraft count in the scenario to the airspace reconfiguration threshold of 22 aircraft is used to estimate the average air traffic control resource utilization. For example, if the average sector aircraft count in the sector was eleven, the average control resource utilization of the sector would be $50 \%$.

As mentioned earlier, however, because traffic was rerouted around the weather cells while maintaining nominal traffic load, some sectors were bound to exceed the threshold of 22 aircraft. In these occurrences, the average aircraft count over the average control resource is assumed to be captured by $F_{i}$, and the maximum average control resource utilization is capped at $100 \%$. Let $U_{i}$ be the average control resource utilization of airspace configuration $i$, given by

$$
U_{i}=\frac{1}{S} \sum_{k=1}^{S} \frac{n(i, k)}{c}
$$

where,

$S$ is the number of sectors in the airspace;

$n(i, k)$ is average aircraft count in sector $k$ in airspace configuration $i$, with a maximum value of $c$;

$c$ is the sector capacity threshold, in number of aircraft.

\section{Airspace Quality Metrics}

\section{Airspace Similarity}

A previous study of airspace reconfiguration impact on air traffic controller workload indicated that a decrease in similarity between the original and reconfigured airspace was related to an increase in controller workload during the airspace reconfiguration [15,16]. The similarity between airspace configuration $i$ and the original was calculated as a similarity distance, $D_{i}$, where larger distance indicates less similarity. Equation (12) from Ref. 17 was used to calculate $D_{i}$, given by

$$
D_{i}=\sum_{k=1 . . S} h\left(k_{i}, O\right)
$$

where, 
$h\left(k_{i}, O\right)$ is the Hausdorff distance [18] between sector $k$ in airspace configuration $i$ and a set of the original sectors $O$;

$S$ is the number of sectors in the airspace;

\section{Number of Flights with Short Dwell Time}

Previous studies on airspace design indicated that increased number of flights with short dwell time in a sector was related to increased controller workload $[15,16]$. Let $T_{i}$ be the average number of flights with short dwell time in airspace configuration $i$, given by

$$
T_{i}=\frac{1}{S} \sum_{k=1}^{S} a(i, k)
$$

where,

$S$ is the number of sectors in the airspace;

$a(i, k)$ is the number of flights with short dwell time (less than three minutes) in sector $k$ in airspace configuration $i$.

\section{Distance between Traffic Crossing Points and Airspace Boundary}

Studies also indicated that a decrease in the average distance between traffic crossing points and airspace boundary was related to an increase in air traffic controller workload $[15,16]$. Let $X_{i}$ be the average traffic crossing point's distance to airspace boundary in airspace configuration $i$, given by

$$
X_{i}=\frac{1}{S} \sum_{k=1}^{S} d(i, k)
$$

where,

$S$ is the number of sectors in the airspace;

$d(i, k)$ is the average distance between traffic crossing points to boundary of sector $k$ in airspace configuration $i$.

\section{Results}

This section summarizes some of the relevant subjective results from the human-in-the-loop simulation, namely the final participant choice in the algorithm selection process and their subjective acceptability ratings. Also, the objective results from the benefits and airspace quality metrics are presented and discussed. The hypothesis is that the objective metrics will correlate with the user selections as well as the subjective ratings.

\section{Participant Selection and Acceptability Feedback}

The results showed that in the 4-sector scenarios, the participants preferred MV (5 out of 8 selections), while in the 7-sector scenarios, it was split between SF and CGS (4 and 3 out of 8 selections, respectively) with a slight bias towards SF. Table 1 summarizes the algorithm-generated airspace designs that were selected by the participants.

Table 1: Participant Selection of Configurations Designed by Algorithms

\begin{tabular}{|c|c|c|c|c|}
\hline & P1 & P2 & P3 & P4 \\
\hline $\begin{array}{c}\text { 4-Sector } \\
\text { Scenario A }\end{array}$ & SF & MV & MV & MV \\
\hline $\begin{array}{c}\text { 4-Sector } \\
\text { Scenario B }\end{array}$ & MV & DAU & CGS & MV \\
\hline $\begin{array}{c}\text { 7-Sector } \\
\text { Scenario A }\end{array}$ & SF & SF & CGS & CGS \\
\hline $\begin{array}{c}\text { 7-Sector } \\
\text { Scenario B }\end{array}$ & SF & DAU & CGS & SF \\
\hline
\end{tabular}

This data is also supported by participant ratings on the acceptability of each algorithm-generated airspace designs. At the end of each simulation run, participants were asked to rate the acceptability of each of the four algorithm-generated configurations from 1 to 6 (Not At All Acceptable = 1, Completely Acceptable $=6)$. The following figure compares the acceptability ratings between the algorithms (Figure 3) $\left(\mathrm{M}_{\mathrm{DAU} 4 \mathrm{~S}}=2.5 ; \mathrm{M}_{\mathrm{CGS} 4 \mathrm{~S}}=3.5 ; \mathrm{M}_{\mathrm{SF} 4 \mathrm{~S}}=3.3 ; \mathrm{M}_{\mathrm{MV} 4 \mathrm{~S}}=\right.$ 4.3; $\mathrm{M}_{\mathrm{DAU} 7 \mathrm{~S}}=2.6 ; \mathrm{M}_{\mathrm{CGS7S}}=4.1 ; \mathrm{M}_{\mathrm{SF7S}}=4.6 ; \mathrm{M}_{\mathrm{MV7S}}=$ 2.4). The three most frequently selected algorithms, namely MV in the 4-sector case, and CGS and SF in the 7-sector case, are differentiated by the color red throughout the paper.

As shown in Figure 3, the most acceptable choice in the 4-sector case was found to be MV, which was also the most selected algorithm for that case. Similarly, both CGS and SF were rated high in the 7-sector case as was the case in the participant selections. A repeated measures ANOVA was carried out on the acceptability ratings and yielded significance in the 4 - and 7 -sector cases $\left(F_{4 \mathrm{~S}}(3,18)=4.00\right.$, $\left.p<.05 ; F_{7 \mathrm{~S}}(3,21)=12.14, p<.001\right)$. This data 
confirms that participants did in fact select the algorithm that was most acceptable of the set. (N.B. One participant's ratings were excluded from the analysis of the 4-sector case because of a missing data point.)

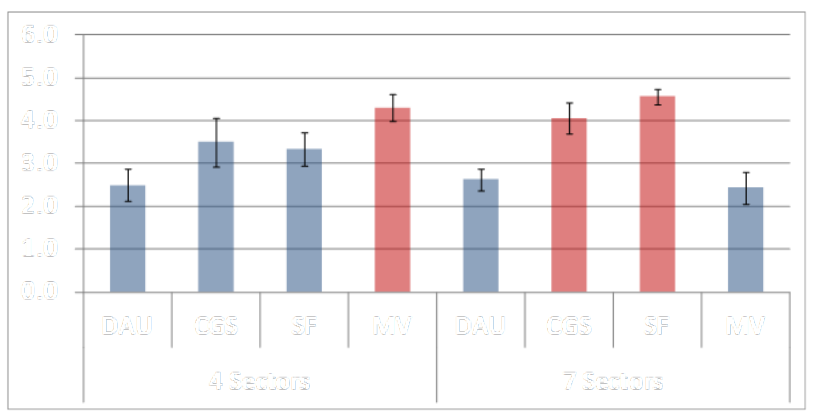

Figure 3: Subjective Acceptability Ratings of Algorithms - Not At All Acceptable (1) to Completely Acceptable (6)

\section{Benefits Metrics}

The total number of removed flights are computed and plotted in Figure 4. The figure shows that the selected algorithms in both cases resulted in the least amount of aircraft that needed to be removed to maintain the threshold of 22 aircraft, suggesting that participants selected airspace designs that would minimize the number of aircraft to be rerouted or delayed.

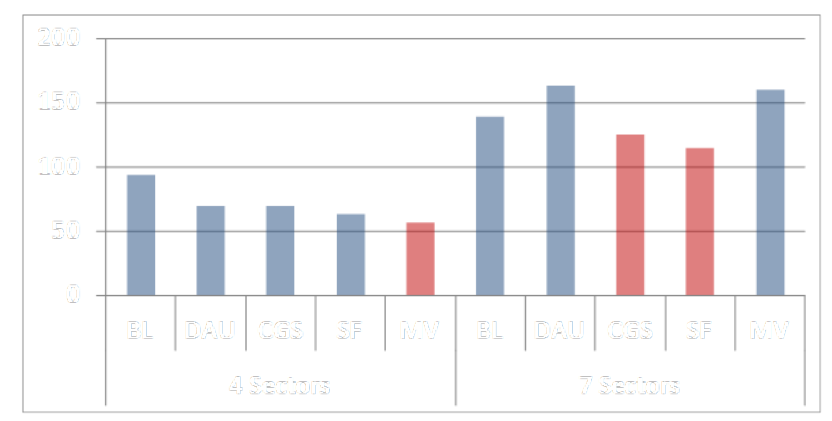

Figure 4: Total Number of Removed Flights

In both the 4- and 7-sector cases, there was a significant difference in the number of removed flights between the algorithms $\left(\chi_{4 \mathrm{~S}}^{2}(4)=11.17, p\right.$ $\left.<.05 ; \chi_{7 \mathrm{~s}}^{2}(4)=12.67, p<.05\right)$. In the 4-sector case, all four algorithms resulted in an overall reduction in the total number of removed aircraft compared to a baseline configuration with no airspace change $\left(\mathrm{T}_{\mathrm{BL}}\right.$ $\left.=94 ; \mathrm{T}_{\mathrm{DAU}}=70 ; \mathrm{T}_{\mathrm{CGS}}=70 ; \mathrm{T}_{\mathrm{SF}}=63 ; \mathrm{T}_{\mathrm{MV}}=57\right)$, suggesting that all algorithms achieved the benefits of reducing the traffic overload by changing the airspace. However, in the 7-sector case, DAU and MV actually increased the totals, thereby performing worse than the original baseline configuration, and CGS and SF $\left(\mathrm{T}_{\mathrm{BL}}=139 ; \mathrm{T}_{\mathrm{DAU}}=163 ; \mathrm{T}_{\mathrm{CGS}}=125 ; \mathrm{T}_{\mathrm{SF}}\right.$ $=115 ; \mathrm{T}_{\mathrm{MV}}=160$ ), suggesting that only CGS and SF algorithms achieved the benefits.

As for the air traffic control resource utilization metric, all algorithms on average produced higher control resource utilization rates in both the 4- and 7sector cases with respect to the original configuration (Figure 5), with the exception of MV which in the 7sector case did not increase the utilization with respect to the baseline configuration $\left(\mathrm{M}_{\mathrm{BL} 4 \mathrm{~S}}=72.2 \%\right.$; $\mathrm{M}_{\mathrm{DAU} 4 \mathrm{~S}}=75.3 \% ; \mathrm{M}_{\mathrm{CGS} 4 \mathrm{~S}}=75.6 \% ; \mathrm{M}_{\mathrm{SF} 4 \mathrm{~S}}=74.2 \%$; $\mathrm{M}_{\mathrm{MV} 4 \mathrm{~S}}=74.6 \% ; \mathrm{M}_{\mathrm{BL} 7 \mathrm{~S}}=82.1 \% ; \mathrm{M}_{\mathrm{DAU} 7 \mathrm{~S}}=82.9 \%$; $\left.\mathrm{M}_{\mathrm{CGS7S}}=86.3 \% ; \mathrm{M}_{\mathrm{SF7S}}=86.3 \% ; \mathrm{M}_{\mathrm{MV7S}}=75.9 \%\right)$. For this metric, we did not have enough data to run statistical tests. However, the general trend of the data, especially for the 7-sector case, supports the hypothesis that airspace designs with higher control resource utilization correlates with user selections.

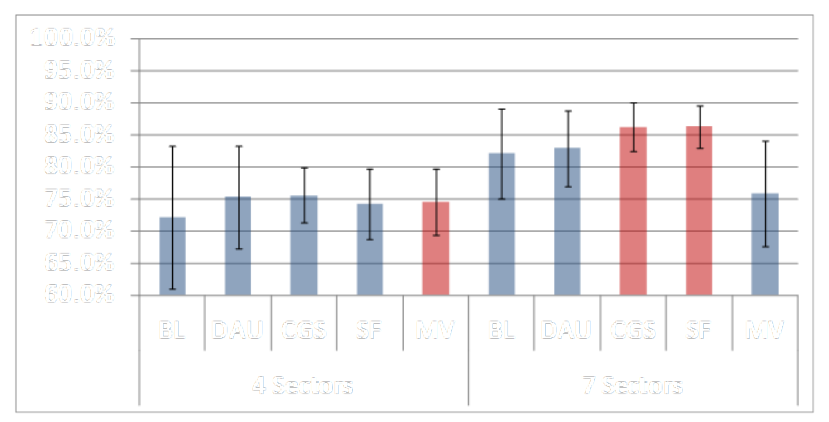

Figure 5: Average Air Traffic Control Resource Utilization

Another way of looking at this data is to compare only the sectors that exhibited the lowest utilization rate. Having a high minimum implies that the algorithm was able to better redistribute the traffic imbalance across the sectors in the test area. Figure 6 illustrates each algorithm's lowest utilization rate, and one can notice that even the worst utilization rates for each algorithm did better than the baseline counterpart in both 4- and 7-sector cases $\left(\mathrm{Min}_{\mathrm{BL} 4 \mathrm{~S}}=11.1 \% ; \operatorname{Min}_{\mathrm{DAU} 4 \mathrm{~S}}=34.9 \% ; \operatorname{Min}_{\mathrm{CGS} 4 \mathrm{~S}}\right.$ $=48.5 \% ; \operatorname{Min}_{\mathrm{SF} 4 \mathrm{~S}}=52.5 \% ; \operatorname{Min}_{\mathrm{MV} 4 \mathrm{~S}}=54.4 \%$; $\operatorname{Min}_{\mathrm{BL} 7 \mathrm{~S}}=9.4 \% ; \operatorname{Min}_{\mathrm{DAU} 7 \mathrm{~S}}=22.9 \% ; \operatorname{Min}_{\mathrm{CGS} 7 \mathrm{~S}}=$ 52.7\%; $\left.\operatorname{Min}_{\mathrm{SF7S}}=63.6 \% ; \operatorname{Min}_{\mathrm{MV} 7 \mathrm{~S}}=12.0 \%\right)$. 


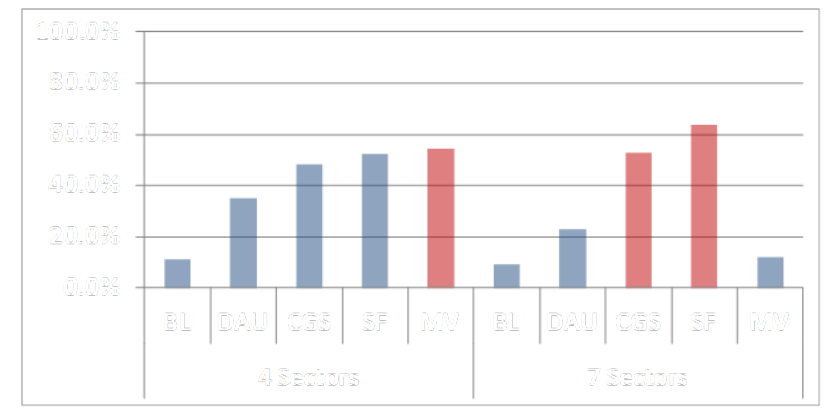

\section{Figure 6: Lowest Air Traffic Control Resource} Utilization Rates

When the algorithms were compared to each other, the selected algorithms were also the ones with the highest minimum in the utilization rate. As shown in Figure 6, MV had the highest minimum utilization rate in the 4-sector case, while both CGS and SF had higher minimum utilization rates than others in the 7 sector case.

\section{Airspace Quality Metrics}

Similarity distance indicates how similar the new airspace configuration is to the original baseline configuration. Shorter similarity distance indicates greater similarity and longer distance indicates less similarity. As shown in Figure 7, DAU generated configurations with the shortest distance from the baseline configuration. On the other hand, MV generated the greatest similarity distance, which, implies that it had the greatest deviation from the original baseline configuration. A repeated measures ANOVA revealed significance in both $4-$ and 7sector cases $\left(F_{4 \mathrm{~S}}(3,21)=15.1, p<.001 ; F_{7 \mathrm{~S}}(3,39)=\right.$ 18.59, $p<.001)$.

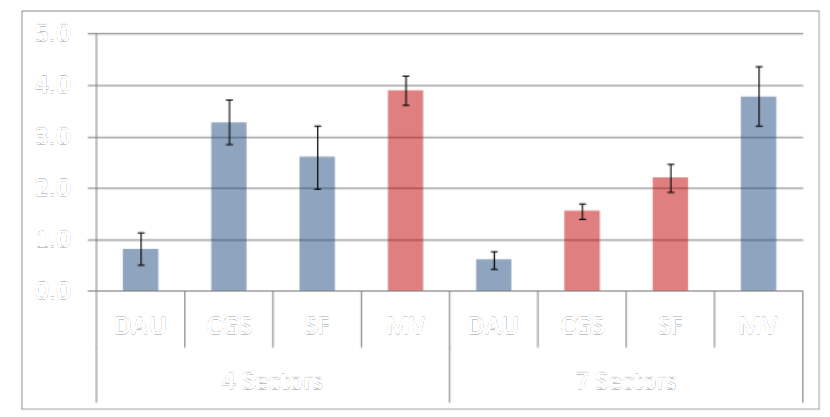

Figure 7: Average Similarity Distance from the Baseline Configuration

Another method to gauge airspace similarity is to examine how each algorithm affected airspace volume at a sector level. A substantial change in sector volumes should be congruent with greater similarity distance, and vice versa. In fact, Figure 8 shows the resulting average volume of unchanged and gained/lost airspace in solid and translucent colors, respectively, and the result parallels that of the similarity distance metric. DAU had the smallest changes in volume while $\mathrm{MV}$ had the greatest changes, in both 4- and 7-sectors. The volume changes incurred by CGS and SF also mirror the distance metric. (N.B. Since the test airspace is bound by the outer bounds of the test sectors as shown in Figure 1, the sum of the two averages is constant.)

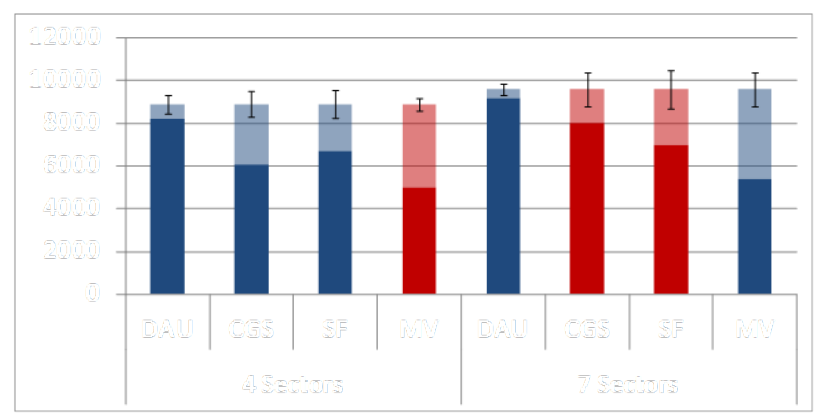

Figure 8: Average Volumes of Unchanged (Solid) and Gained/Lost (Translucent) Airspace (in Cubic Nautical Miles)

When the similarity data is compared to the participants' selections, the results suggest that participants did not pick the airspace designs that maximized the similarities (i.e., minimized the similarity distance), even though such design would have minimized the controller workload during the airspace configuration change. In fact, participants chose MV in the 4-sector case, which had the greatest similarity distance. In the 7-sector case, they chose SF and CGS which had the second and third largest distance. The implications of these results are discussed in the Discussion section.

Another airspace quality metric is the short dwell time for aircraft traversing through a sector. Airspace configurations with short dwell times (defined here as less than three minutes) were considered to be a bad design. As shown in Figure 9, DAU had the highest number of flights with short dwell times in both 4- and 7-sector cases. Figure 2 illustrates that most of DAU configurations had unconventionally shaped sectors with sharp angled edges, such as "panhandles" and "nook and crannies" 
that would have contributed to a number of flights with short dwell times.

Although a set of chi-square tests revealed that there was a strong effect of the algorithms on the total number of short dwell flights, in both 4- and 7 sector cases $\left(\chi_{4 \mathrm{~S}}^{2}(4)=41.96, p<.001 ; \chi_{7 \mathrm{~s}}^{2}(4)=\right.$ $156.18, p<.001)$, this metric did not correlate with the user selections.

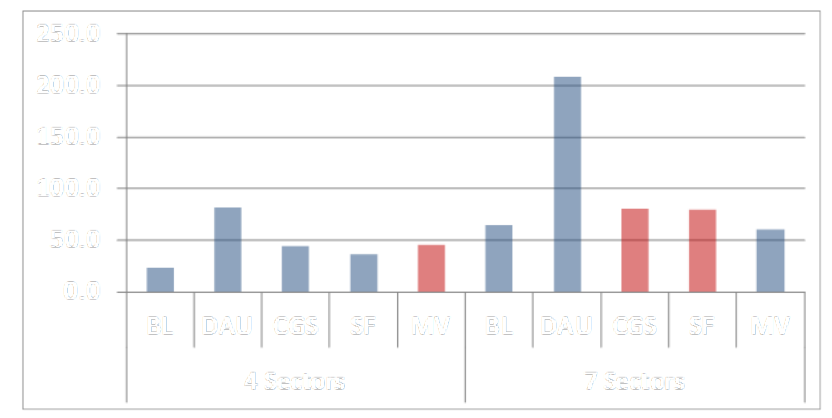

Figure 9: Total Number of Short Dwell Flights

Lastly, the average distance between traffic crossing points and the sector boundaries are shown in Figure 10. Greater distance between traffic crossing points and the sector boundaries are considered to be good airspace design. As shown in Figure 9, MV in the 4-sector case had its crossing points farthest from its boundaries, supporting our hypothesis. SF in the 7-sector case also resulted in the greatest distance to the boundaries. F-tests revealed that the metric was statistically significant only in the 4-sector case $\left(\mathrm{M}_{\mathrm{BL} 4 \mathrm{~S}}=14.0 ; \mathrm{M}_{\mathrm{DAU} 4 \mathrm{~S}}=\right.$ 12.3; $\mathrm{M}_{\mathrm{CGS} 4 \mathrm{~S}}=12.6 ; \mathrm{M}_{\mathrm{SF} 4 \mathrm{~S}}=13.9 ; \mathrm{M}_{\mathrm{MV} 4 \mathrm{~S}}=15.4$; $\mathrm{M}_{\mathrm{BL} 7 \mathrm{~S}}=12.9 ; \mathrm{M}_{\mathrm{DAU7S}}=11.9 ; \mathrm{M}_{\mathrm{CGS7S}}=10.7 ; \mathrm{M}_{\mathrm{SF7S}}=$ $\left.12.5 ; \mathrm{M}_{\mathrm{MV7S}}=14.5\right)\left(F_{4 \mathrm{~S}}(4,28)=2.95, p<.05 ;\right.$ $F_{7 \mathrm{~S}}(4,48)=0.95$, n.s. $)$.

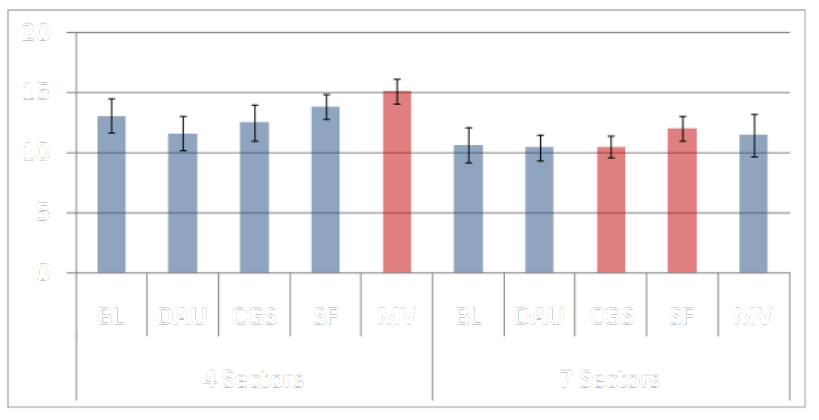

Figure 10: Average Traffic Crossing Points Distance to Sector Boundary (in Nautical Miles)

\section{Discussion}

Two broad categories of metrics, i.e., ones that capture the benefits of the airspace configuration change and the others that capture the "quality" of the airspace designs, were hypothesized to correlate with the participants' ratings. The results showed that participants' selection correlated mainly with the benefits metrics, while airspace quality metrics did not play a big role. Table 2 summarizes whether or not each metric was found to be consistent with the paper's hypothesis.

Table 2: Summary of Metrics' Consistency with Respect to the Hypothesis

\begin{tabular}{|c|c|c|c|}
\hline & & 4-Sector & 7-Sector \\
\hline \multirow{2}{*}{ 串 } & Removed Flights & Consistent & Consistent \\
\hline & $\begin{array}{l}\text { Resource } \\
\text { Utilization }\end{array}$ & Consistent & Consistent \\
\hline \multirow{3}{*}{ 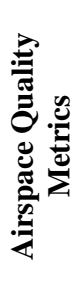 } & $\begin{array}{l}\text { Similarity } \\
\text { Distance }\end{array}$ & $\begin{array}{c}\text { Not } \\
\text { Consistent }\end{array}$ & $\begin{array}{c}\text { Not } \\
\text { Consistent }\end{array}$ \\
\hline & $\begin{array}{l}\text { Short Dwell } \\
\text { Time Flights }\end{array}$ & $\begin{array}{c}\text { Not } \\
\text { Consistent }\end{array}$ & $\begin{array}{c}\text { Not } \\
\text { Consistent }\end{array}$ \\
\hline & $\begin{array}{l}\text { Traffic Crossing } \\
\text { Points Distance }\end{array}$ & Consistent & $\begin{array}{l}\text { Partially } \\
\text { Consistent }\end{array}$ \\
\hline
\end{tabular}

Although different algorithms were chosen in different scenarios, there was a common thread among the chosen configurations. The data suggest that the chosen configurations were consistent with the airspace designs with most benefits. Higher rated airspace designs managed the peak aircraft count below the threshold better while distributing the traffic more evenly across the test sectors. Although the average airspace utilization rate showed similar findings, the comparison of the sectors with lowest utilization rate across the different algorithms was more indicative of the selected airspace designs.

The data were less consistent for the airspace quality metrics. If higher airspace design quality was the main factor in participants' choice of airspace designs, then higher quality should correlate with higher participants' ratings. For the similarity distance metric, the data suggests that greater similarity distance correlated with the user selections 
in the 4-sector case. This result can be explained by Jung et al. [5], who postulated that an increase in similarity distance is often related to an increase in the benefit. Therefore, participants might have selected the airspace designs with greater benefits, ignoring the cost of larger airspace changes (i.e., greater similarity distances), presumably because benefit gains are more important for the tasks that they are performing. In the 7-sector case, the same pattern holds if MV is ignored, which seemed to have resulted in greater dissimilarity without generating greater benefits.

The short dwell time flights did not show a simple relationship with the participants' airspace acceptability ratings. However, the metric might indicate the features of bad sector designs that the users will reject. For example, DAU had significantly more aircraft count with short dwell times than other algorithms, which was reflected in the participants' acceptability ratings despite close similarities between the new and the original configurations. It is possible that participants will select airspace with short dwell times up to a point if the benefits exist, but they may reject airspace designs with excessive number of aircraft with short dwell times. Further studies are needed to validate this conjecture.

Lastly, the traffic crossing points distance to sector boundary metric partially supported our hypothesis. While the 4-sector case resulted in greater distance for the selected airspace designs the results were less prominent in the 7-sector case.

Based on the results, it seemed that the participants' were inherently focused on the benefits metrics rather than the airspace quality metrics in their selection of the airspace design and their airspace acceptability ratings. A possible explanation could be that participants were given the task of managing the traffic demand, which biased their selection process to consider the benefits of managing the traffic over the costs of implementing "bad" airspace design. In general, there seems to be an inherent tension between benefits and costs in selecting the right airspace configuration.

In addition, airspace quality metrics can be divided into two categories. The similarity distance metric is designed to assess controller workload during airspace reconfiguration, while the short dwell time flights and traffic crossing points distance metrics are designed to assess controller workload after airspace reconfiguration. In other words, both categories of metrics are related to the costs of airspace reconfiguration. However, information to examine the airspace quality, such as graphical representation of traffic crossing points over the sectors, was not given. It is then perhaps safe to assume that the participants were mainly focused on the benefits, and not the costs.

Furthermore, the airspace designs were considered for around one hour time duration, which could be mapped easily to maximize the benefits of managing the traffic situation in a similar time window. Unlike long term solutions which need to focus more on the airspace quality metrics given that costs associated to the controller workload cannot be sustained, short term solutions, on the other hand, can concentrate more on the benefits metrics. And given that the premise of the study was that the new airspace configuration would only be in effect for short term, perhaps the participants had deemed the short term benefits to outweigh the short-term costs. This then can explain why the hypothesis is supported only by the two benefits metrics.

A different context, such as selecting an airspace design needs to be optimal for a day, may yield different results. Traffic can vary significantly over longer time duration, making it difficult to derive an airspace design that can maximize the benefits over the entire duration, leading them to weigh more on the airspace quality than the benefits metrics.

This paper has taken an initial analysis of user selection criteria for "good" airspace design using airspace benefits and quality metrics. Follow-up studies are needed to further explore and evaluate the different operational contexts in which the airspace benefits and quality metrics can differentially impact the user selection of airspace designs.

\section{References}

[1] Kopardekar, P., K. Bilimoria, B. Sridhar, 2007, Initial concepts for Dynamic Airspace Configuration, $7^{\text {th }}$ Aviation Technology, Integration and Operations (ATIO) Seminar, Belfast, Northern Ireland.

[2] Homola, J., P.U. Lee, C. Brasil, H.E. Lee, M. Mainini, 2011, Human-in-the-Loop Investigation of Airspace Design, AIAA Guidance, Navigation, and Control Conference and Exhibit, Portland, OR. 
[3] Lee, P.U., C. Brasil, J. Homola, A. Kessell, H.E. Lee, M. Mainini, T. Prevot, 2011, Benefits and Feasibility of the Flexible Airspace Management Concept: A Human-in-the-Loop Evaluation of Roles, Procedures, and Tools, USA/Europe Air Traffic Management R\&D Seminar, Berlin, Germany.

[4] Homola, J., P.U. Lee, T. Prevot, H.E. Lee, A. Kessell, C. Brasil, N. Smith, 2010, A Human-in-theLoop Exploration of the Dynamic Airspace Configuration Concept, AIAA Guidance, Navigation, and Control Conference and Exhibit, Toronto, Canada.

[5] Jung, J., F.L. Lai, S. Zelinski, 2011, Benefits of Mid-Term Flexible Airspace Management in Presence of Weather, AIAA 2011-6690, AIAA Guidance, Navigation, and Control Conference, Portland, OR.

[6] Prevot, T., P. Lee, T. Callantine, J. Mercer, J. Homola, N. Smith, E. Palmer, 2010, Human-in-theLoop Evaluation of NextGen Concepts in the Airspace Operations Laboratory, AIAA Modeling and Simulation Technologies Conference and Exhibit, Toronto, Canada.

[7] Klein, A., M. Rogers, H. Kaing, 2008, Dynamic FPAs: A New Method for Dynamic Airspace Configuration, Integrated Communications Navigation and Surveillance Conference, Bethesda, MD.

[8] Yousefi, A., 2010, Optimal Airspace Partitioning: Cell Based Optimization Approach, INFORMS Journal of Transportation Science, October, 2010.

[9] Sabhnani, G., J. Mitchell, A. Yousefi, 2010, Flow Conforming Operational Airspace Sector Design, $10^{\text {th }}$ AIAA Aviation Technology, Integration, and Operations Conference, Fort Worth, TX.

[10] Brinton, C., S. Pledgie, 2008, Airspace Partitioning using Flight Clustering and Computational Geometry. $27^{\text {th }}$ Digital Avionics Systems Conference, Saint Paul, MN.

[11] Kopardekar, P., S. Magyarits, 2003, Measurement and Prediction of Dynamic Density. $5^{\text {th }}$ USA/Europe Air Traffic Management R\&D Seminar, Budapest, Hungary.
[12] Xue, M., 2010, Three-Dimensional Sector Design with Optimal Number of Sectors, AIAA Guidance, Navigation, and Control Conference and Exhibit, Toronto, Canada.

[13] Xue, M., 2009, Airspace Sector Redesign Based on Voronoi Diagrams, Journal of Aerospace, Computing, Information, and Communication, AIAA, Vol.6, No.12, pp.624-634.

[14] Meyn, L., R. Windhorst, K. Roth, D. VanDrei, G. Kubat, V. Manikonda, S. Roney, G. Hunter, A. Huang, G. Couluris, 2006, Build 4 of the Airspace Concept Evaluation System, AIAA 2006-6110, AIAA Modeling and Simulation Technologies Conference and Exhibit, Keystone, CO.

[15] Lee, P.U., T. Prevot, J. Homola, H.E. Lee, A. Kessell, N. Smith, 2010, Sector Design and Boundary Change Considerations for Flexible Airspace Management, AIAA 2010-9376, $10^{\text {th }}$ AIAA Aviation Technology, Integration, and Operations Conference, Fort Worth, TX.

[16] Federal Aviation Administration, 2005, Airspace Management Handbook, Version 2.2, MITRE Corporation.

[17] Yousefi, A., R. Hoffman, M. Lowther, B. Khorrami, H. Hackney, 2009, Trigger Metrics for Dynamic Airspace Configuration, AIAA 2009-7103, $9^{\text {th }}$ AIAA Aviation Technology, Integration, and Operation Conference, Hilton Head, SC.

[18] Grégoire, N., M. Bouillot, 1998, Hausdorff Distance Between Convex Polygons, McGill University, Montreal, Canada.

\section{Acknowledgements}

This work was funded by NASA's Airspace Systems Program and NextGen-Airspace Project. The authors would like to thank the participants in this study, members of the algorithm development teams as well as MACS' developers.

30th Digital Avionics Systems Conference October 16-20, 2011 